\title{
The glass transition in a model silica glass: evolution of the local structure
}

\author{
P. Jund and R. Jullien \\ Laboratoire des Verres - Université Montpellier 2 \\ Place E. Bataillon Case 069, 34095 Montpellier France
}

\begin{abstract}
We use molecular dynamics simulations and the Voronoï tessellation to study the geometrical modifications as a function of temperature in a model silica glass. The standard deviation of the cell volumes, which is a measure of the local density fluctuations, decreases with decreasing temperature, as if it would like to vanish at zero temperature. This evolution towards an ordered state is frozen out at the glass transition and consequently an amorphous sample is obtained at low temperature. This structural freezing following upon the glass transition is noticeable in all the other geometric characteristics of the Voronoï cells and a possible interpretation in terms of geometrical frustration is proposed.
\end{abstract}

PACS numbers: 61.43.Fs, 02.70.Ns, 61.43.Bn, 64.70.Pf

Typeset using REVTEX 


\section{INTRODUCTION}

Silica is a common material which is of great importance in chemistry, geology and industrial applications. It is also a prototype of a network forming glass. All these reasons explain why it has been the topic of a great amount of studies. Nevertheless many features of this typical "strong" glass need still to find a satisfactory explanation. For example the origin of the First Sharp Diffraction Peak (FSDP) is still controversial (Elliott 1991; Gaskell and Wallis 1996; Fayos, Bermejo, Dawidowski, Fischer and Gonzalez 1996). The origin (and the connection to the FSDP) of the so-called Boson peak remains the topic of many studies, both experimental and theoretical (Shuker and Gammon 1970, Martin and Brenig 1974; Akkermans and Maynard 1985; Buchenau, Zhou, Nücker, Gilroy and Phillips 1988; Malinovsky, Novikov, Parshin, Sokolov and Zemlyanov 1990; Novikov and Sokolov 1991; Sokolov, Kisliuk, Soltwisch and Quitmann 1992; Börjesson, Hassan, Swenson, Torell and Fontana 1993; Gurevich, Parshin, Pelous and Schober 1993; Achibat, Boukenter and Duval 1999; Schirmacher and Wagener 1993; Bermejo, Criado and Martinez 1994; Terki, Levelut, Boissier and Pelous 1996). With the development of the computing speed a new type of studies has emerged in the past decade which can be called "computer experiments". Indeed numerical simulations have been used to study the vibrational spectrum (Jin, Vashista, Kalia and Rino 1993; Taraskin and Elliot 1997a; Horbach, Kob and Binder 1997; Taraskin and Elliott 1997b; Guillot and Guissani 1997) or the structural characteristics (Woodcock, Angell and Cheeseman 1976; Holender and Morgan 1991; Della Valle and Andersen 1992; Servalli and Colombo 1993; Nakano, Bi, Kalia and Vashista 1993; Sarnthein, Pasquarello and Car 1995; Vollmayr, Kob and Binder 1996) of various model silica glasses. Within this framework we present in this paper a classical molecular dynamics study essentially focused on the evolution of the local structure in such a system.

The first step in that kind of endeavor is to choose the interparticle potential: we decided to use the two-body potential proposed by van Beest et al. (van Beest, Kramer and van Santen 1990). Indeed a study of the influence of the quenching rate on the properties of 
amorphous silica has shown that this potential based on ab initio parameters gives excellent results compared to experimental data both for the structural characteristics (Vollmayr et al. 1996) and the vibrational properties (Taraskin and Elliott 1997b) of vitreous silica, even if, in simulations the quenching rates are always faster than in real systems. Similarly to what has been done earlier in the case of high pressure silica samples (Rustad, Yuen and Spera 1991) we combine the molecular dynamics with the Voronoï tessellation scheme in order to have a better insight into the structural evolution of the system during the quenching procedure. We want to know if and how the glass transition leaves a "signature" in the geometrical characteristics of the Voronoï cells. We recall that the Voronoï cell attached to a particle is an extension, for disordered systems, of the Wigner-Seitz cell and gives information on the local structure around this particle.

Our results as a function of temperature show the structural freezing consecutive to the glass transition in all the geometric characteristics of the Voronoï cells. We suggest that the structural freezing below $T_{g}$ is mainly a consequence of the impossibility to build an ideal structure as a consequence of the so-called "geometrical frustration" (Kleman and Sadoc 1979; Kleman 1983, 1985, 1989; Sadoc and Mosseri 1997).

In section II a description of the simulations will be given. We present our results in section III and discuss them in section IV. Finally in section V we present our conclusions.

\section{SIMULATIONS}

If one wants to perform realistic simulations on silica glasses the major point is to choose an interaction potential which gives reasonable results compared to experimental data. Several choices are today possible, but in the last years one of the most successful classical potentials is the so-called BKS potential developed by van Beest et al. (van Beest et al. 1990). Though designed originally from the crystalline phases of silica, it has been shown that it also describes very well the structural (Vollmayr et al. 1996) and vibrational (Taraskin and Elliott 1997b) properties of amorphous silica. 
The functional form of the pairwise BKS interaction between two particles $i$ and $j$ is given by

$$
U\left(r_{i j}\right)=\frac{q_{i} q_{j} e^{2}}{r_{i j}}+A_{i j} \exp \left(-B_{i j} r_{i j}\right)-\frac{C_{i j}}{r_{i j}^{6}}
$$

where $r_{i j}$ is the interparticle distance, $e$ the charge of an electron and the parameters $A_{i j}$, $B_{i j}$ and $C_{i j}$ are fixed as follows: $A_{S i O}=18003.7572$ and $A_{O O}=1388.773 \mathrm{eV} ; B_{S i O}=4.87318$ and $B_{O O}=2.76 \AA^{-1} ; C_{S i O}=133.5381$ and $C_{O O}=175.0 \mathrm{eV} \AA^{6}$. Note that except for the Coulomb interaction $\left(q_{S i}=2.4\right.$ and $\left.q_{O}=-1.2\right)$ there is no interaction between Si atoms. This original form contains an unphysical property at short distances since it diverges to minus infinity. To overcome this drawback which is especially annoying at high temperature, we have added a short range repulsive term $\left(\sim 1 / r_{i j}^{40}\right)$ which insures that the potential goes to infinity at small interatomic distances and is practically equivalent to the original potential for $r_{i j} \geq 1.2 \AA$ (Si-O interaction), and $r_{i j} \geq 1.6 \AA$ (O-O interaction) without introducing any artificial energy barrier.

The Coulomb interactions were computed using the Ewald summation method (Ewald 1921; Allen and Tildesley 1990) with a characteristic constant $\kappa=6.5 / L$, where $L$ is the cubic box size, and considering $501 \mathrm{k}$-vectors in reciprocal space $(|k| \leq 6 \times 2 \pi / L)$. These values insure that the potential energy is obtained with a relative error smaller than $5 \times 10^{-4 \%}$. No cut-off was used for the pairwise interaction.

We performed molecular dynamics simulations for microcanonical systems containing 216 silicon and 432 oxygen atoms confined in a cubic box of edge length $L=21.48 \AA$, which corresponds to a mass density of $\approx 2.18 \mathrm{~g} / \mathrm{cm}^{3}$ very close to the experimental value of $2.2 \mathrm{~g} / \mathrm{cm}^{3}$. Periodic boundary conditions were used to limit surface effects. In order to insure energy conservation even at high temperature a timestep of 0.7 fs was necessary. This value is substantially lower than the one used in previous studies (1.6 fs (Vollmayr et al. 1996) or 1.0 fs (Taraskin and Elliott 1997b)) which may be due to our "conservative" potential correction. The 4-th order Runge-Kutta algorithm was used to integrate the equations of motion. The glass configurations were obtained by quenching well equilibrated initial liquid samples 
obtained by melting $\beta$-cristobalite crystals at a temperature around $7000 \mathrm{~K}$. After full equilibration of the liquid ( $\approx 40000$ timesteps), the system was cooled to zero temperature at a quench rate of $2.3 \times 10^{14} \mathrm{~K} / \mathrm{s}$ which was obtained by removing the corresponding amount of energy from the total energy of the system at each iteration. Due to computer time limitations this cooling rate is rather fast but it has the advantage compared to other procedures involving either stepwise cooling (Vollmayr et al. 1996) or temperature dependent rates (Taraskin and Elliott 1997b), to be linear and continuous all along the quenching procedure. At several temperatures during the quenching process the configurations (positions and velocities) were saved. Each configuration was used to start a constant-energy molecular dynamics calculation during which the temperature was recorded as a function of time. The temperature was in all cases remarkably stable and only slight relaxation effects could be observed. Nevertheless to avoid transient configurations we allowed for each temperature and for each sample, 10000 relaxation steps followed by 50000 supplemental time steps (for a total simulation time of $42 \mathrm{ps}$ ) during which all the calculations were done. Apart from the calculation of standard quantities (radial pair distribution function, mean square displacement) we included also in our molecular dynamics code a Voronoï tessellation scheme similar to the one that we have developed for monocomponent soft-sphere glasses (Jund, Caprion and Jullien 1997a; 1997b). This scheme has been modified to take into account several types of atoms and thus it permits to follow the local structure around the silicon atoms and the oxygen atoms as a function of temperature during the quenching procedure. Here the Voronoï cell is always defined as being the region of space closer to a given atom center than to any other and no dissymetry between the two components has been introduced as it is the case in the "navigation map" procedure (Gellatly and Finney 1982). The Voronoï cell characteristics as well as all the other quantities have been averaged over samples obtained from 5 independent starting configurations in order to improve the statistics of the results. The whole simulation lasted for more than 3 million timesteps which were run on 4 nodes of an IBM/SP2 parallel computer. 


\section{RESULTS}

As said earlier this potential has already been used in other studies of amorphous silica and since we did not modify the BKS parameters our results concerning the radial pair distribution (Vollmayr et al. 1996; Taraskin and Elliott 1997b) or the diffusion constant (Guillot and Guissani 1997) are exactly identical to the referenced results and therefore we do not come back to these standard results here. Our aim is to localize the glass transition temperature $T_{g}$ through the study of the structural characteristics (via the Voronoï tessellation) of our model silica system. Nevertheless a straightforward way of determining $T_{g}$ is to monitor the potential energy versus the temperature, as has already been done for another model silica glass (Della Valle and Andersen 1992). The evolution of the average potential energy per particle versus the temperature is shown in Fig. 1. With increasing temperature, the potential energy as well as the standard deviation increases as expected. Nevertheless one can observe an acceleration of this increase between 3000 and $4000 \mathrm{~K}$, corresponding to the passage from a solid to a liquid behavior. Due to the fast cooling rate the value of $T_{g}$ is much higher than the experimental value (1446 K (Angell 1988)) but it is coherent with the value of $\approx 3500 \mathrm{~K}$ obtained from the fit of $T_{g}$ versus the quenching rate proposed by Vollmayr et al. (Vollmayr et al. 1996). Note that we observe a non negligible increase of the potential energy in the glass phase contrarily to what was obtained by Della Valle et al (Della Valle and Andersen 1992, Fig. 3) who used a direct minimization procedure after the quench to investigate the "inherent structures" (Stillinger and Weber 1982). Here we let the system evolve freely after the quench and it seems that even at low temperatures structural relaxation occurs. Note also that for such a fast cooling rate the structure of the resulting silica might very well be slightly different than the one observed experimentally, since it is known that to get the most "ideal" glass, one should use the slowest quenching rate which does not produce crystallization (Kauzman 1948; Jund, Caprion and Jullien 1997c). However we recall that previous authors (Vollmayr et al. 1996; Taraskin and Elliott 1997b), using the same potential with a quenching rate of the same order of magnitude as ours, were 
able to reproduce several physical properties of real silica.

Once we know approximately the value of $T_{g}$ (to our purpose this level of accuracy is sufficient) we can tackle the study of the geometrical characteristics of the Voronoï cells in order to follow the local structure as a function of temperature. All the characteristics of the cells have been obtained (surface, number of faces, number of edges, etc...) but we want to discuss here only some representative quantities. The first one is the variation of the volume of the Voronoï cells. This variation is represented in Fig.2 for the silicon (a) and the oxygen (b) atoms together with the corresponding standard deviations (c). With decreasing temperature the volume of the silicon cell decreases while the volume of the oxygen cell increases (these opposite variations are a consequence of our constant-volume calculations). Again a change of behavior is visible and corresponds to a slowing down of the evolution below the glass transition temperature. An even more striking behavior is observed in Fig.2c where the standard deviation $\sigma_{V}$ is plotted as a function of temperature. This is a quantity of physical interest since it measures the local density fluctuations around the particles. For both types of atoms, $\sigma_{V}$ decreases with decreasing temperature as if it would like to tend to zero at $T=0 \mathrm{~K}$ but then below $4000 \mathrm{~K}$ this trend is stopped and finally $\sigma_{V}$ saturates around non-zero values characteristic of spatial disorder. This is a direct observation of the low-temperature saturation of the density fluctuations which is a signature of the glass transition. Even though not reported here, exactly the same behavior can be observed for the evolution of the surface of the Voronoï cells as a function of temperature.

To investigate further the structural evolution during the quench, we looked at the angle distributions. To numerically determine the bond angle O-Si-O (resp. Si-O-Si), we determine for each Si (resp. O) atom the two nearest O (resp. Si) atoms and we calculate the angle between the two corresponding segments Si-O (resp. O-Si), the result being averaged over all the Si (rep. O) atoms. First we studied the tetrahedral O-Si-O angle which should be ideally equal to $109^{\circ} .47$ in a perfect tetrahedron. As can be seen in Fig.3a, this angle varies between $110^{\circ} .5$ at low temperature and $117^{\circ}$ at $7000 \mathrm{~K}$, with a slight change of behavior around $T_{g}$. The same behavior is observed for the corresponding standard deviation (Fig.3c 
full circles) which is quite small and decreases when $T$ decreases. This shows firstly that with increasing temperature the $\mathrm{SiO}_{4}$ tetrahedra survive even in the liquid phase but become more and more distorted and secondly that the glass transition does not strongly affect the local environment around the silicon atoms. On the contrary the glass transition is more clearly visible in the angle $\mathrm{Si}-\mathrm{O}-\mathrm{Si}$, which measures the relative position and orientation of two neighboring $\mathrm{SiO}_{4}$ tetrahedra, as can be seen in Fig.3b. With decreasing temperature within the liquid phase, the Si-O-Si angle increases and seems to converge towards $180^{\circ}$ (a least square quadratic fit of the four points in the liquid phase gives an extrapolated value of $175^{\circ}$ ), but again below $4000 \mathrm{~K}$ this increase slows down and finally the angle converges towards a value close to $150^{\circ}$, a value coherent with previous simulations (Vollmayr et al. 1996; Taraskin and Elliott 1997b), but slightly higher than the value $144^{\circ}$ found in X-ray diffraction experiments (Neuefeind and Liss 1996). The decrease of this angle is coherent with the views of a densifying network with increasing temperature this densification taking place around the oxygen atoms. Also since it measures the relative orientation between two neighboring tetrahedra, this decrease corresponds to a decrease of the effective volume of the oxygen atoms with increasing temperature and since we work at constant volume it implies an expansion of the silicon volume, which is indeed the behavior observed in Fig. 2. Concerning the standard deviation of the Si-O-Si angle represented in Fig.3c (open circles) it increases rapidly in the liquid phase (above $4000 \mathrm{~K}$ ) while in the glass phase this increase is more slow. This again is an illustration of the structural freezing below $T_{g}$.

Another quantity which reflects the glass transition is the effective coordination number, $z$, which is in fact the average number of faces of the Voronoï cells. This quantity should be considered with some care since, generally, it does not correspond to the true "chemical" coordination number. To give an example, in $\beta$-cristobalite the Voronoï cell of the silicon atoms is a tetrahedron $(z=4)$ while the Voronoï cell of the oxygen atoms is the polyhedron represented in Fig. 4 with $z=8$. In the Voronoï "sense" the nearest neighbors of an oxygen atom in $\beta$-cristobalite are two silicon atoms (represented by the triangular faces in Fig. 4), and six oxygen neighbors (represented by the pentagonal faces in Fig. 4). Such a cell is 
a truncated double-tetrahedron and is, in fact, highly "degenerate": some of its edges are shared by four neighboring cells (instead of three usually). An infinitely small random perturbation of the atomic positions $\left(\approx 10^{-2} \AA\right)$ is sufficient to create new extra-small triangular faces and the coordination number of the oxygen atoms flips to $z \approx 19.7$ while the coordination of the silicon atoms remains close to 4 . This could be avoided by doing a smoothed Voronoï tessellation which excludes small faces with an area smaller than $10 \%$ of the largest cell face (Rustad et al. 1991) but we have rather chosen not to use this technique and analyze raw data. The variation of $z$ as a function of temperature is represented in Fig. 5a for the silicon atoms and Fig. 5b for the oxygen atoms. The glass transition can again clearly be identified: for the silicon atoms, $z$ increases with increasing temperature with a slope that is more important above $4000 \mathrm{~K}$. For the oxygen atoms, $z$ is approximately constant in the glass phase and then decreases for temperatures above $T_{g}$. It should be noted that the relative variation of $z$ between 0 and $7000 \mathrm{~K}$ is small, especially for the oxygen atoms. In the liquid phase, with decreasing temperature, the local structure around the silicon atoms evolves towards a perfect tetrahedral arrangement $(z=4)$ and then converges to a value higher than 4 in the glass phase due again to the structural freezing below $T_{g}$.

\section{DISCUSSION}

All these results can be discussed in the light of the conclusions drawn for the analogous geometrical analysis performed in a monoatomic soft-sphere glass (Jund et al. 1997a, 1997b). In that case, the system tries to reach an icosahedral arrangement (with dodecahedral Voronoï cells) when the temperature is lowered down from the liquid phase, but, since such an arrangement can not be realized at large distances in the regular three-dimensional space, the system gets frozen in a glass phase below a characteristic glass temperature. Such geometrical frustration effects were in that case the consequence of the degeneracy between

the face-centered-cubic (FCC) and the hexagonal-close-packed (HCP) structures (Sadoc and Mosseri 1997). 
Since all the standard deviations presented here seem to go to zero with decreasing temperature, when extrapolated from the liquid phase, it is reasonable to assume that in the case of silica also a $T=0$ unreachable ideal local structure exists. It is also reasonable to assume that such an ideal arrangement corresponds to a perfect tetrahedral order for the four oxygens bounded to a given silicon atom, as it is for almost all of the known crystalline structures of silica. This assumption is supported by the behavior of $\mathrm{z}$, which tries to extrapolate to 4 when $T$ is lowered from the liquid phase, and it is not incompatible with our results for the variation of the angle O-Si-O with temperature (see Fig.3a). Even if this angle does not show a major change of behavior at $T_{g}$, an extrapolated value of $109 .^{\circ} 47$ at $T=0$ is not inconsistent with the reported data above $T_{g}$. Moreover, since the angle $\mathrm{Si}$-O-Si seems to extrapolate to $180^{\circ}$, one can imagine that the ideal structure is made of tetrahedral units, like the $s p_{3}$ coordination of carbon where the silicon atoms would be located at the carbon places and oxygen atoms located in the middle of the $\mathrm{C}-\mathrm{C}$ bonds (see Fig. 6a). Indeed the tendency to build such a local structure should result from the form of the potential. In particular the tetrahedral arrangement of the oxygens around a silicon atom results from a combination of the Si-O attraction and the repulsion between the oxygens. The tendency to align the Si-O-Si bridges between neighboring tetrahedra is more subtle however, since the long range nature of the ionic part of the potential certainly plays a role.

It is interesting to notice that, among all the known crystalline structures of silica, two particular structures (at least) fully satisfy these criteria, namely the $\beta$-cristobalite and the tridymite structures. In these two structures the above defined tetrahedral units are stacked with sequences $\mathrm{ABCABC} . .($ Fig.6b) and ABABAB...(Fig.6c), respectively, like in FCC and HCP structures. In fact these structures can be simply built from FCC and HCP structures, by adding to the original structure another one shifted by a fourth of the diagonal of the cubic cell (in the FCC case) and by $3 / 8$ of the $c$-axis of the hexagonal cell (in the HCP case). They correspond respectively to the diamond and wurtzite structures of carbon. When these two structures are considered with the same density, they have exactly the same Si-O distance $d_{S i O}$, and therefore, in the two cases the Voronoï cells for the silicon atoms are 
regular tetrahedra with the same volume $V_{S i}=\sqrt{3} d_{S i O}^{3}$. The volume of the oxygen cells, $V_{O}$, is also the same and therefore the two structures are characterized by the same $\mathrm{Si} / \mathrm{O}$ volume ratio $R=V_{S i} /\left(2 V_{O}\right)=9 / 55=0.164$, independent on the density. In Fig.7 we have plotted $R=V_{S i} /\left(2 V_{O}\right)$ as a function of $T$, as calculated from our simulations, and reported the value $R=0.164$ at $T=0$ (open circle). When decreasing the temperature from the liquid phase $R$ decreases as if it would like to reach a value quite close to (or even lower than) 0.164. Therefore, one could conclude that may be the ideal structure that the system would try to realize when lowering temperature would be a mixture of tridymite and cristobalite phases. In fact this is not a satisfying solution since these two phases can very well arrange themselves under the form of crystallites separated by dislocations or stacking faults, and therefore crystallization should actually occur in our system.

One can find a solution by pursuing the analogy with soft sphere glasses or hard sphere packings for which also two degenerate ground state arrangements FCC and HCP exist. In that case the system gets "frustrated" by trying to reach a local icosahedral structure (Jund et al. 1997a, 1997b; Jullien, Jund, Caprion and Quitmann 1996) which does not exist in the flat regular space. Similarly to these model systems, the frustration can be resolved by considering in our case a curved space with positive curvature, namely the sphere $S_{3}$ (Sadoc and Mosseri 1997). Consider a regular tetrahedron with a silicon atom in the middle and oxygen atoms at the centers of the faces. This tetrahedral unit contains one silica molecule. One can exactly tile an $S_{3}$ space with 600 tetrahedra like this, and the resulting $\mathrm{SiO}_{2}$ structure (which contains 600 silicons and 1200 oxygens) satisfies all the local requirements defined above. The 120 vertices of the unit tetrahedra are located on the so-called $\{3,3,5\}$ polytope (Sadoc and Mosseri 1997). Note that an important difference with tridymite and cristobalite is that this ideal structure is made of five-membered ..Si-O-... rings, instead of six-membered ones, since there are five unit tetrahedra around a tetrahedron edge in the $\{3,3,5\}$ polytope. It is not trivial to calculate the exact value of the ratio $R$ for this structure but a good approx- 
imation can be obtained by considering the tetrahedron unit in the flat three dimensional space tangent to the curved space at the tetrahedron center. The silicon Voronoï cell, limited by the bisector planes of the $\mathrm{Si}-\mathrm{O}$ bonds, is a tetrahedron of volume $1 / 8$ of the volume of the unit. Therefore the remaining volume for the two oxygen atoms is $7 / 8$ and consequently $R=1 / 7=0.143$. This value is only approximate due to the fact that volumes do not exactly scale as distance-cubes in the curved space $S_{3}$ of positive radius $r_{s}$. An estimation of the error made can be obtained by performing the same reasoning for two concentric spheres of radii $r$ and $r / 2$, for which one knows analytic formulae for their volumes (Sadoc and Mosseri 1997). Performing an expansion in $r / r_{s}$, one gets $R \approx(1 / 7)\left(1+(2 / 35)\left(r / r_{s}\right)^{2} \ldots\right)$ instead of $1 / 7$. Taking for $r$ the radius of the sphere of same volume than the one of the unit tetrahedron (which is the volume of $S_{3}$ divided by 600), this formula gives 0.144 instead of 0.143 . Hence, in view of the roughness of the extrapolations done, such an error can reasonably be neglected.

This value is represented by the open square in Fig.7. It appears that this value corresponds to a better extrapolation of the four points above $4000 \mathrm{~K}$ than the one obtained from tridymite or cristobalite (the second order fit of the four liquid points leads to a value of 0.136). Therefore one is tempted to conclude as in the soft sphere case (Jund et al. 1997a; 1997b): when lowering the temperature from the liquid phase the system evolves as if it would try to build locally such an ideal structure, but since this structure cannot be realized in the regular three dimensional space, the systems gets frozen in a glassy state below $T_{g}$. Obviously, the above interpretation should be considered as a suggestion and there are certainly other ways to resolve the frustration. One way is to consider the "polytope $240 "$ described in chapter 2 of the Sadoc and Mosseri book (Sadoc and Mosseri 1997). In that case, one would get six-membered rings. One could also imagine solutions with seven membered rings. The only argument in favor of our simple suggestion is the nice extrapolation of the volume ratio data to 0.143 . We have checked that this ratio is significantly larger for the polytope 240 solution. Definitely more calculations are needed to validate our proposal. In particular a systematic enumeration of the $n$-membered rings as a function of temperature 
would be helpful. Such an enumeration already exists in the literature (Vollmayr et al. 1996) but has been done as a function of quenching rate.

\section{CONCLUSIONS}

With the use of classical molecular dynamics simulations combined with the Voronoï tessellation we have studied the evolution of the local structure around the particles of a model silica glass as a function of temperature. The glass transition temperature, $T_{g}$, obtained from the variation of the potential energy versus the temperature is coherent with the value expected for systems quenched at $2.3 \times 10^{14} \mathrm{~K} / \mathrm{s}$. This transition is clearly visible in the evolution of all the geometric characteristics of the Voronoï cells. The study of the local density fluctuations clearly demonstrates the saturation of these fluctuations below $T_{g}$ which is the usual signature of the glass transition. The study of the tetrahedral O-Si-O angle shows that with increasing temperature the $\mathrm{SiO}_{4}$ tetrahedra survive but become more and more distorted. The variation of the angle Si-O-Si between two corner-sharing tetrahedra is coherent with the views of a densifying network with increasing temperature, this densification happening around the oxygen atoms, while a volume expansion occurs around the silicon particles since we are considering microcanonical ensembles. The different geometric characteristics and in particular the average coordination number evolve with decreasing temperature as if the system would like to reach an ideal structure at $T=0$, which cannot be realized due to geometrical frustration. This evolution is frozen below the glass transition temperature, and finally the system converges towards an amorphous structure.

Our results show that the use of the Voronoï cell characteristics gives not only useful informations on the local structure but can also be used to determine the glass transition unambiguously. This means that even if nothing dramatic happens at the glass transition concerning the local structure, something happens, which is basically a dynamic freezing of the natural evolution of the structure towards an unreachable ideal structure. Of course we

are far away from the timescales used in experiment, but this study, together with others, 
permits to investigate what happens on the microscopic level in an attempt to explain what is observed at the macroscopic level.

\section{ACKNOWLEDGMENTS}

We thank C. Levelut and J. Pelous for helpful discussions. Part of the numerical calcu-

lations were done at CNUSC (Centre National Universitaire Sud de Calcul), Montpellier. 


\section{REFERENCES}

ACHiBAt, T., BOUKEnTER, A. and DUval, E., 1993, J. Chem. Phys., 99, 2046.

AKKERMANS, E. and MAYNARD, R., 1985, Phys. Rev. B, 32, 785.

ALLEN, M.P. and TILDESLEY, D.J., 1990, Computer simulation of liquids, Oxford University Press, New-York.

ANGELL, C.A., 1988, J. Chem. Phys. Solids, 49, 863.

BERMEJO, F.J., CRIADO, A. and MARTINEZ, J.L., 1994, Phys. Lett. A, 195, 236.

BÖRJESSON, F.L., HASSAN, A.K., SWENSON, J., TORELL, L.M. and FONTANA, A., 1993, Phys. Rev. Lett., 70, 1275.

BUCHENAU, U., ZHOU, H.M., NÜCKER, N., GILROY, K.S. and PHILLIPS, W.A., 1988, Phys. Rev. Lett., 60, 1318.

DELla VAlle, R.G. and ANDERSEN, H.C., 1992, J. Chem. Phys., 97, 2682.

ELLIOTT, S.R., 1991, Phys. Rev. Lett., 67, 711.

EWALD, P.P., 1921, Ann. Phys., 64, 253.

FAYOS, R., BERMEJO, F.J., DAWIDOWSKI, J., FISCHER, H.E. AND GONZALEZ, M.A., 1996, Phys. Rev. Lett., 77, 3823.

GASKELL, P.H. AND WALLIS, D.J., 1996, Phys. Rev. Lett., 76, 66.

GEllatly, B.J. and FINNEY, J.L., 1982, J. Non Cryst. Solids, 50, 313.

GUILLOT, B. and GUISSANI, Y., 1997, Phys. Rev. Lett., 78, 2401.

GUREVICH, V.L., PARSHIN, D.A., PELOUS, J., and SCHOBER, H.R., 1993, Phys. Rev. $B, 48,16318$.

HOLEnDER, J.M. and MORGAN, G.J., 1991, J. Phys: CM, 3, 1947.

HORBACH, J., KOB, W. and BINDER, K., 1997, preprint.

JIN, W., VASHISTA, P., KALIA, R.K. and RINO, J.P., 1993, Phys. Rev. B 48, 9359.

JUlliEn, R., JUND, P., CAPRION, D. and QUITMANN, D., 1996, Phys. Rev. E, 54, 6035. 
JUND, P., CAPRION, D. and JULLIEN, R., 1997a, Europhys. Lett., 37, 547.

JUND, P., CAPRION, D. and JULLIEN, R., 1997b, Mol. Sim., 20, 3.

JUND, P., CAPRION, D. and JUllien, R., 1997c, Phys. Rev. Lett., 79, 91.

KLEMAN, M., 1983, J. de Physique (France), 44, L295.

KLEMAN, M., 1985, J. de Physique (France), 46, L723.

KLEMAN, M., 1989, Adv. in Phys., 38, 605.

KLEMAN, M. and SADOC, J.-F., 1979, J. de Physique (France), 40, L569.

MALINOVSKY, V.K., NOVIKOV, V.N., PARSHIN, P.P., SOKOLOV, A.P. and ZEMLYANOV, M.G., 1990, Europhys. Lett., 11, 43.

MARTIN, A. and BRENIG, W., 1974, Phys. Status Solidi B, 64, 163.

NAKANO, A., BI, L., KALIA, R.K. and VASHISTA, P., 1993, Phys. Rev. Lett., 71, 85.

NEUEFEIND, J. and LISS, K.D., 1996, Ber. Buns. Ges. Phys. Chem., 100, 1341.

NOVIKOV, V.N. and SOKOLOV, A.P., 1991, Solid State Commun., 77, 243.

RUSTAD, R., YUEN, D.A. and SPERA, F.J., 1991, Phys. Rev. B 44, 2108.

SADOC, J.F. and MOSSERI, R., 1997, La frustration géométrique, Alea Collection, Eyrolles ed., Saclay, France.

SARnthein, J., PASQUAREllO, A. and CAR, R., 1995, Phys. Rev. Lett., 74, 4682.

SERVALLI, G. and COLOMBO, L., 1993, Europhys. Lett., 22, 107.

SCHIRMACHER, W., and WAGENER, M., 1993, Solid State Commun., 86, 597.

SHUKER, E. and GAMMON, R.W., 1970, Phys. Rev. Lett., 25, 223.

SOKOLOV, A.P., KISLIUK, A., SOLTWISCH, M. and QUITMANN, D., 1992, Phys. Rev. Lett., 69, 1540.

STILlingeR, F.H. and WEBER, T.A., 1982, Phys. Rev. A, 25, 978.

TARASKIN, S.N. and ELLIOTT, S.R., 1997a, Phys. Rev. B 55, 1.

TARASKIN, S.N. and ELLIOTT, S.R., 1997b, Europhys. Lett, 39, 37.

TERKI, F., LeVelut, C., BOissier, M. and PelOus, J., 1996, Phys. Rev. B 53, 2411. VAN BEEST, B.W.H., KRAMER, G.J. and VAN SANTEN, R.A., 1990, Phys. Rev. Lett., 64, 1955. 
VOLlmaYR, K., KOB, W. and BINDER, K., 1996, Phys. Rev. B 54, 15808.

WOODCOK, L.V., ANGELL, C.A. and CHEESEMAN, P., 1976, J. Chem. Phys., 65, 1565. 


\section{FIGURES}

Fig. 1. Average potential energy per particle as a function of temperature.

Fig. 2. Variation of the cell volume versus temperature: (a) silicon; (b) oxygen.

(c) Standard deviation $\sigma_{V}$ versus temperature: •: silicon; o: oxygen.

Fig. 3. Variation as a function of temperature of: (a) $\theta$, the O-Si-O angle, (b) $\phi$, the SiO-Si angle; (c) the standard deviations $\sigma_{\theta}$ and $\sigma_{\phi}$ versus temperature: •: silicon; ०: oxygen.

Fig. 4. The Voronoï cell of an oxygen atom in $\beta$-cristobalite.

Fig. 5. Variation of the effective coordination, $z$, as a function of temperature: (a) silicon atoms; (b) oxygen atoms.

Fig. 6. The ideal tetrahedral unit around a silicon atom (a) is shown together with the trydimite (b) and $\beta$-cristobalite (c) structures viewed from the top. In (b) and (c) two successive layers connected to a single top unit are represented (the positions of the oxygen atoms have been omitted).

Fig. 7. Variation of $R=V_{S i} / 2 V_{O}$ as a function of temperature. ०: value of $R$ obtained in tridymite or $\beta$-cristobalite; $\square$ : value of $R$ obtained in the ideal structure built on $S_{3}$. 
Figure 1

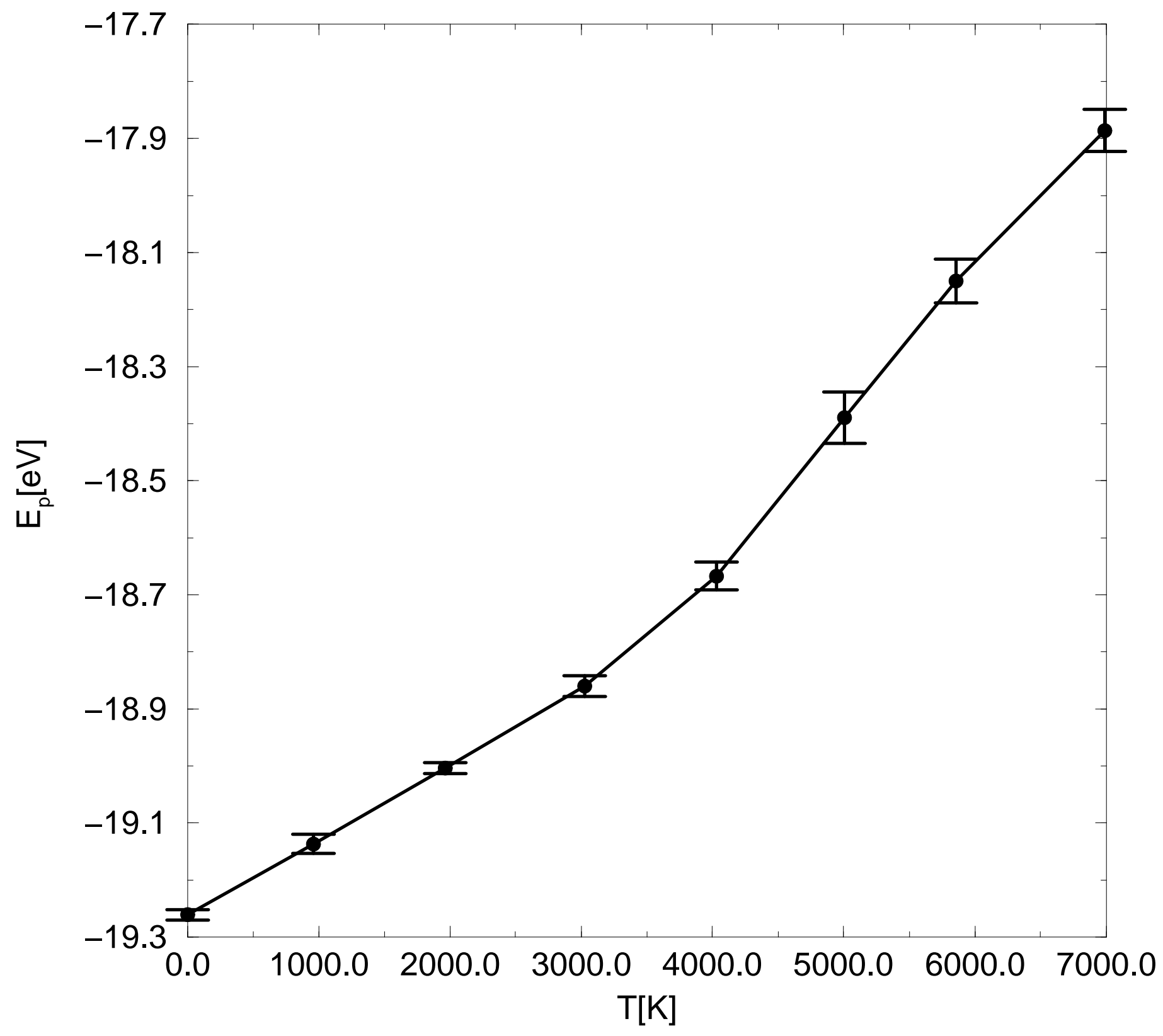


Figure 2

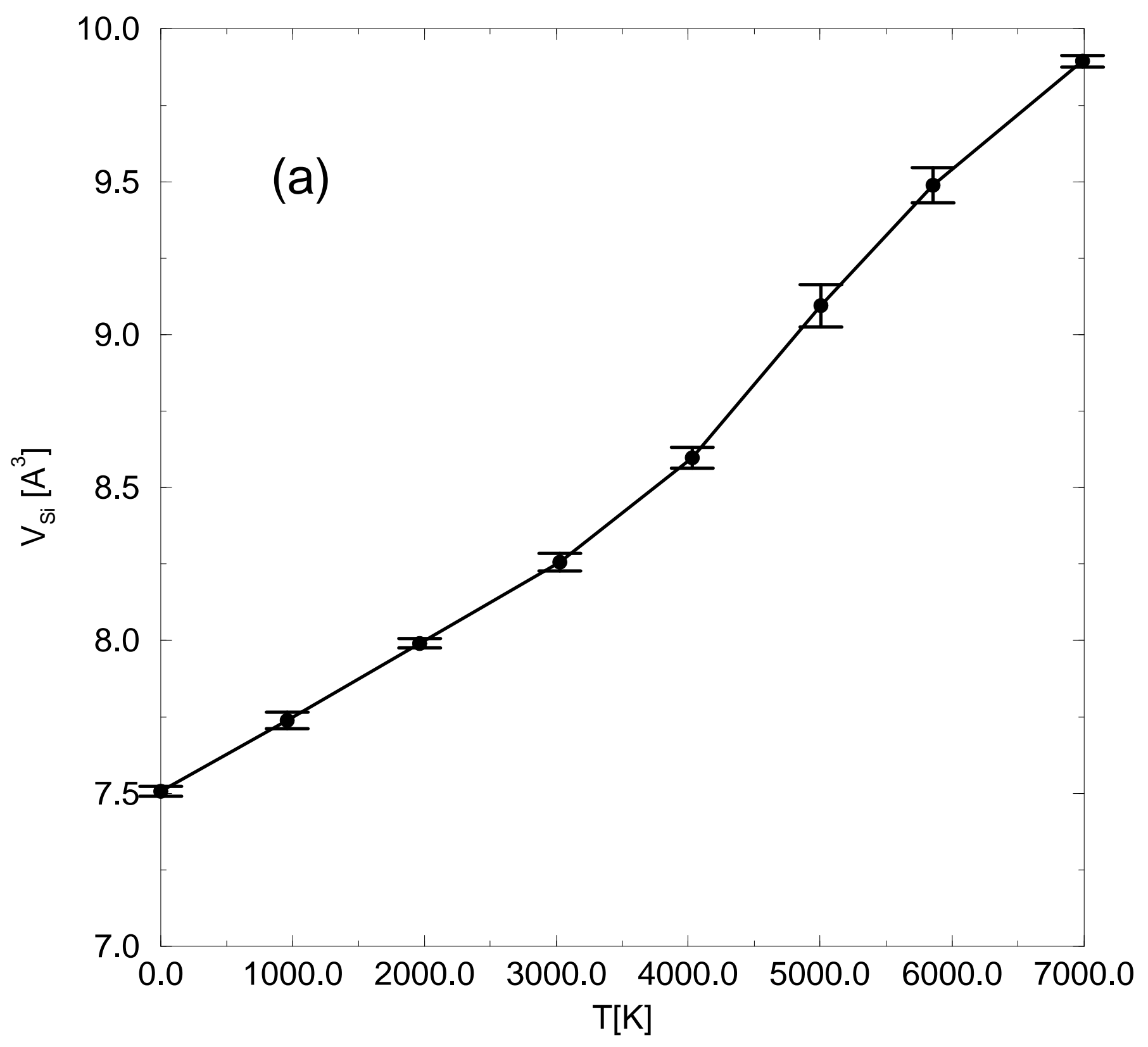




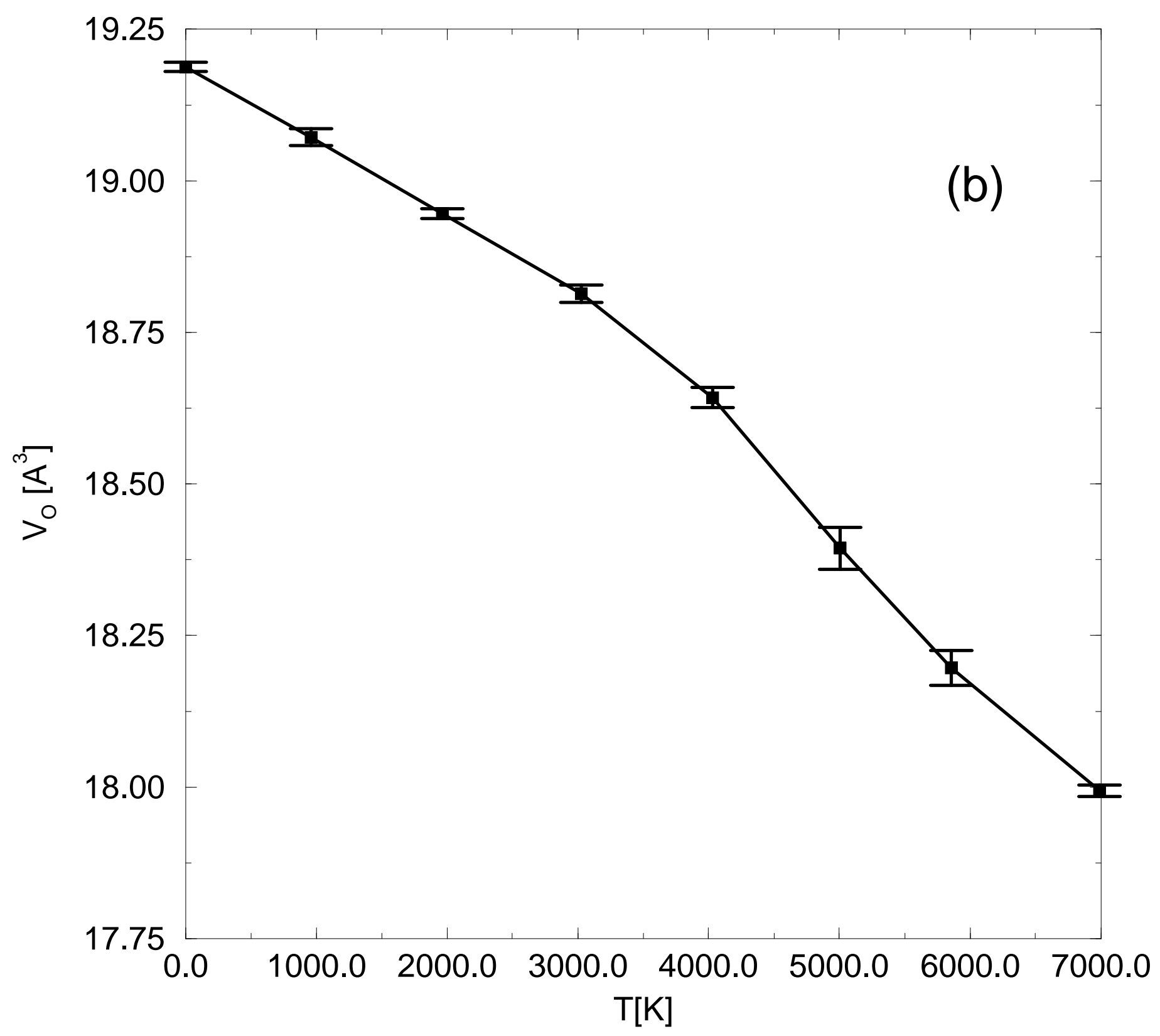




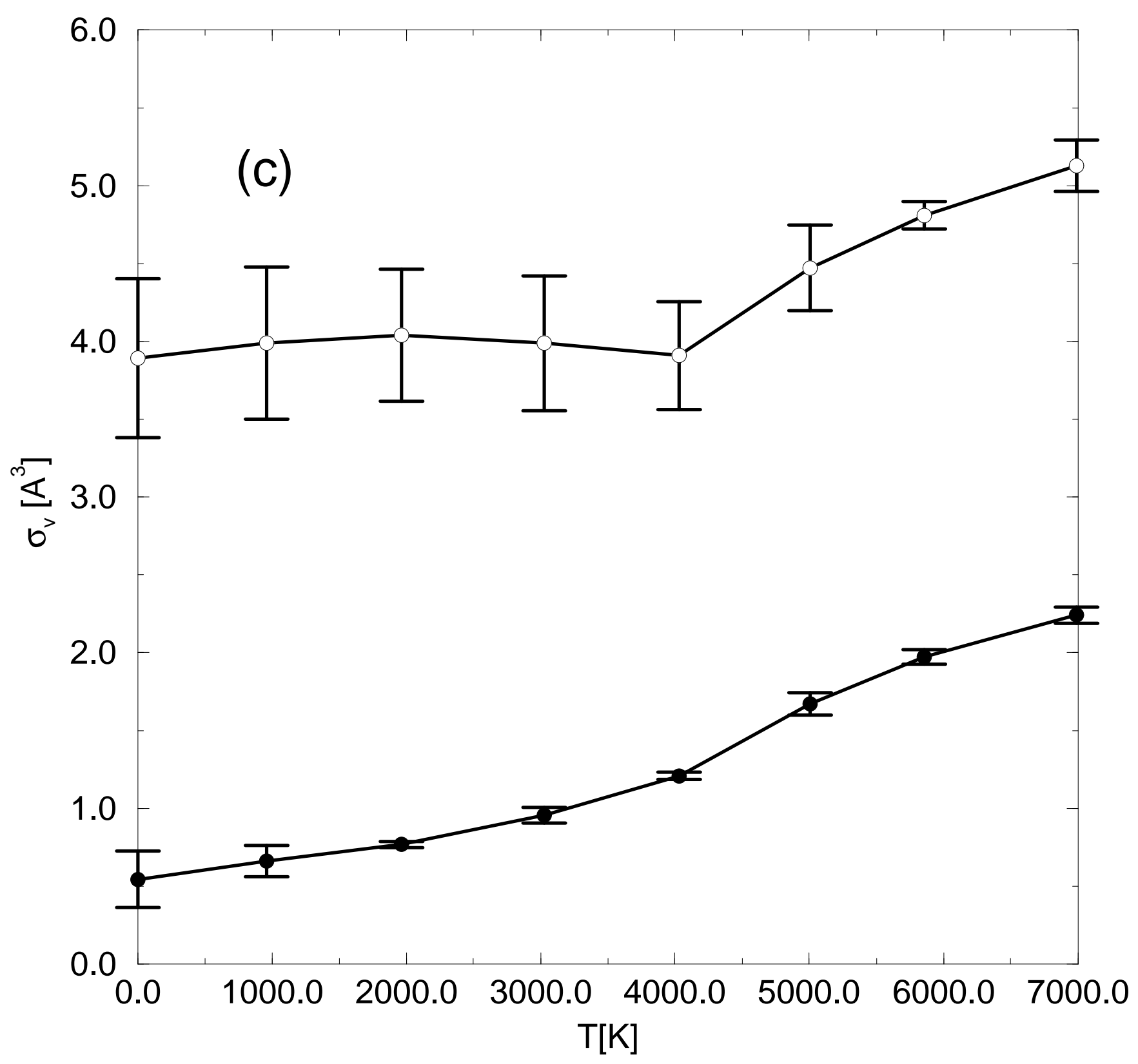




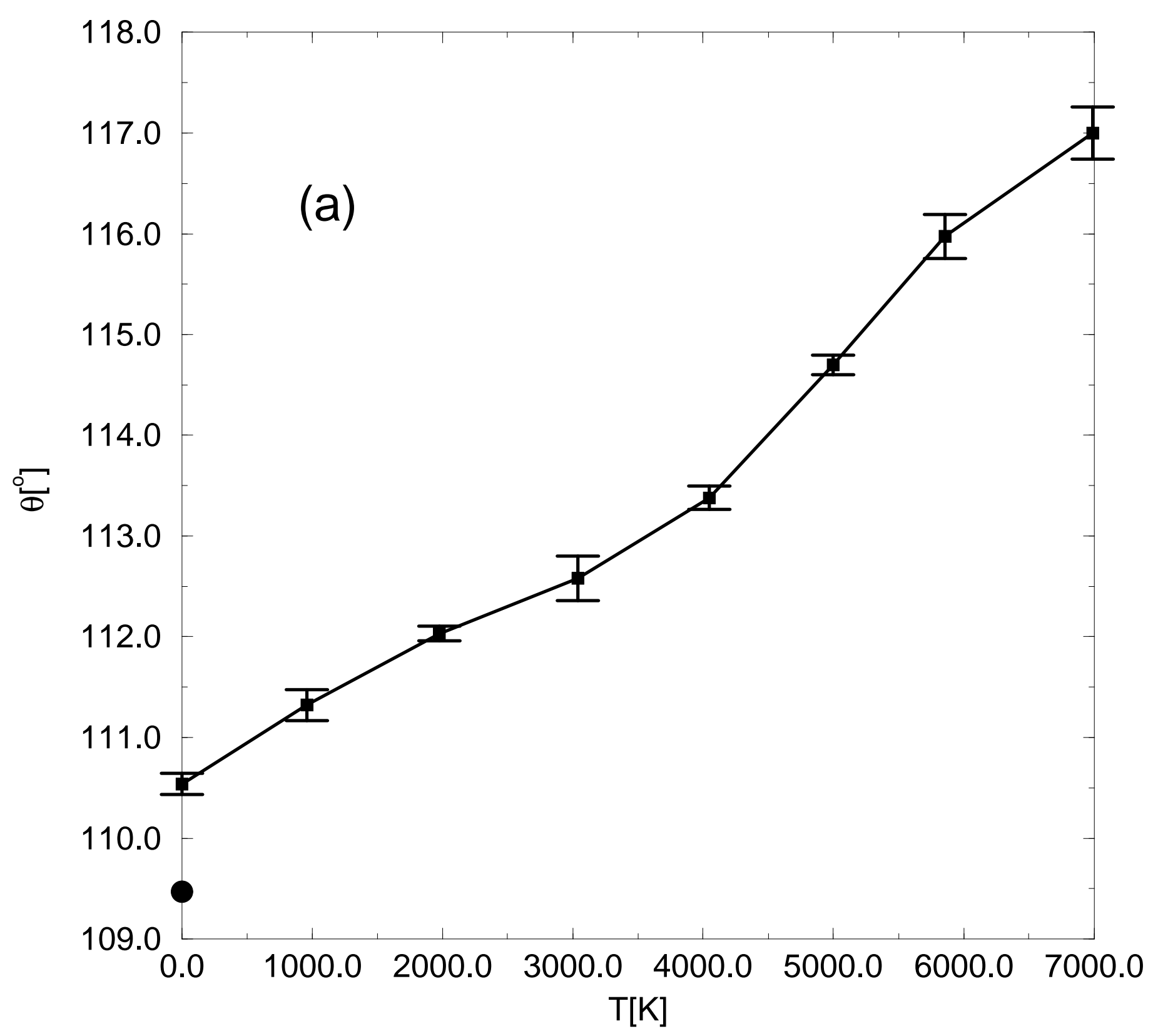




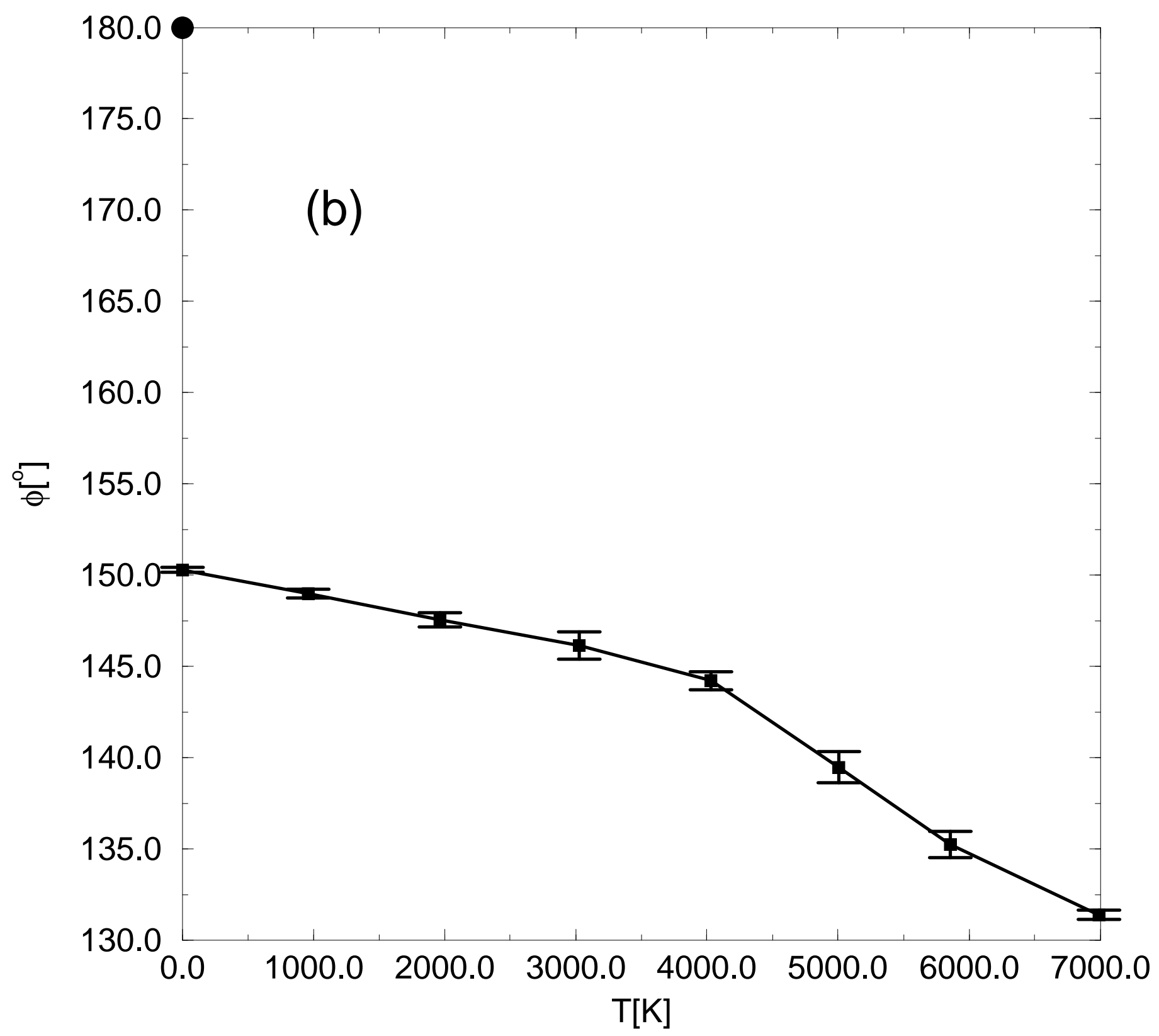




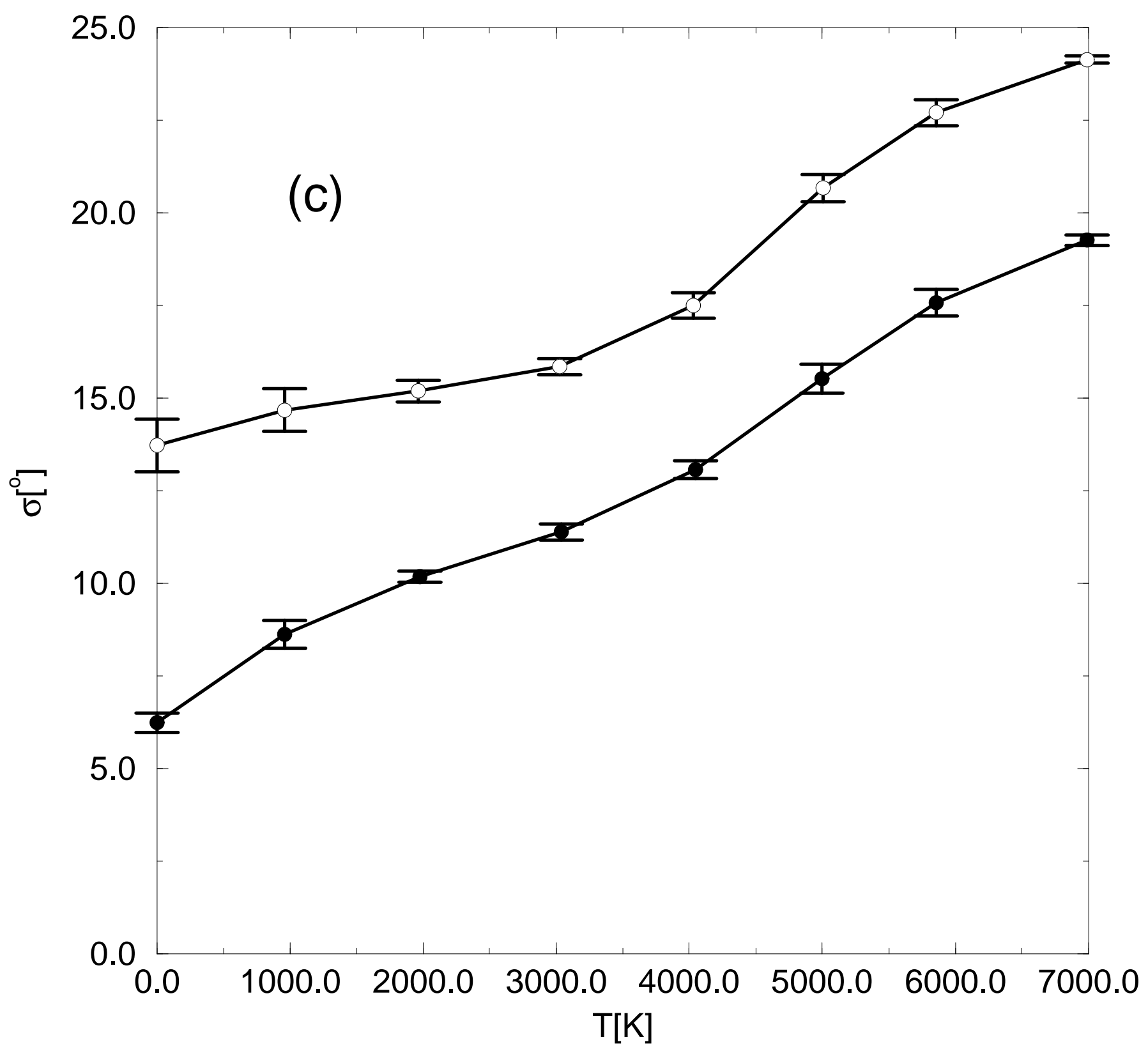




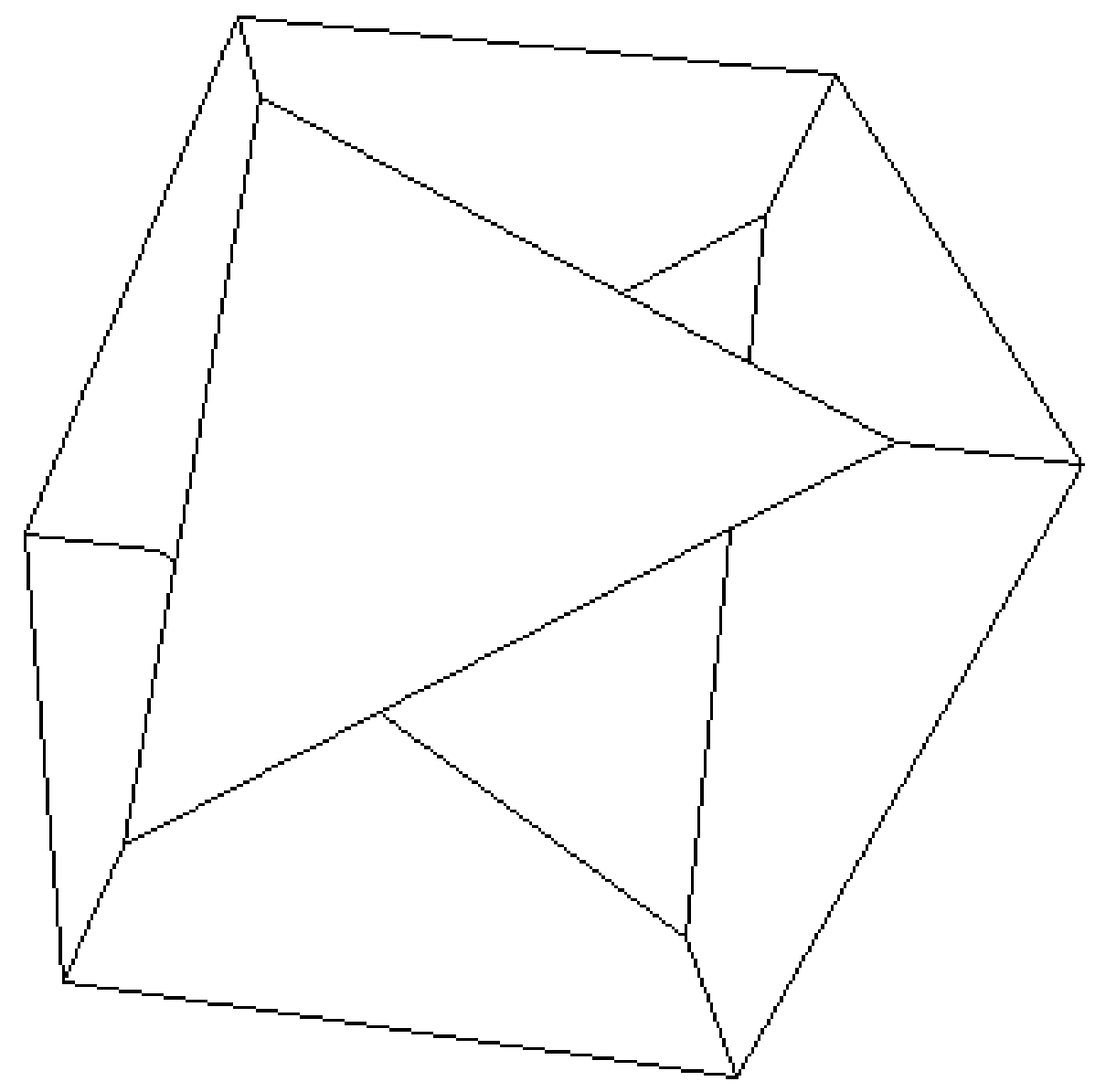




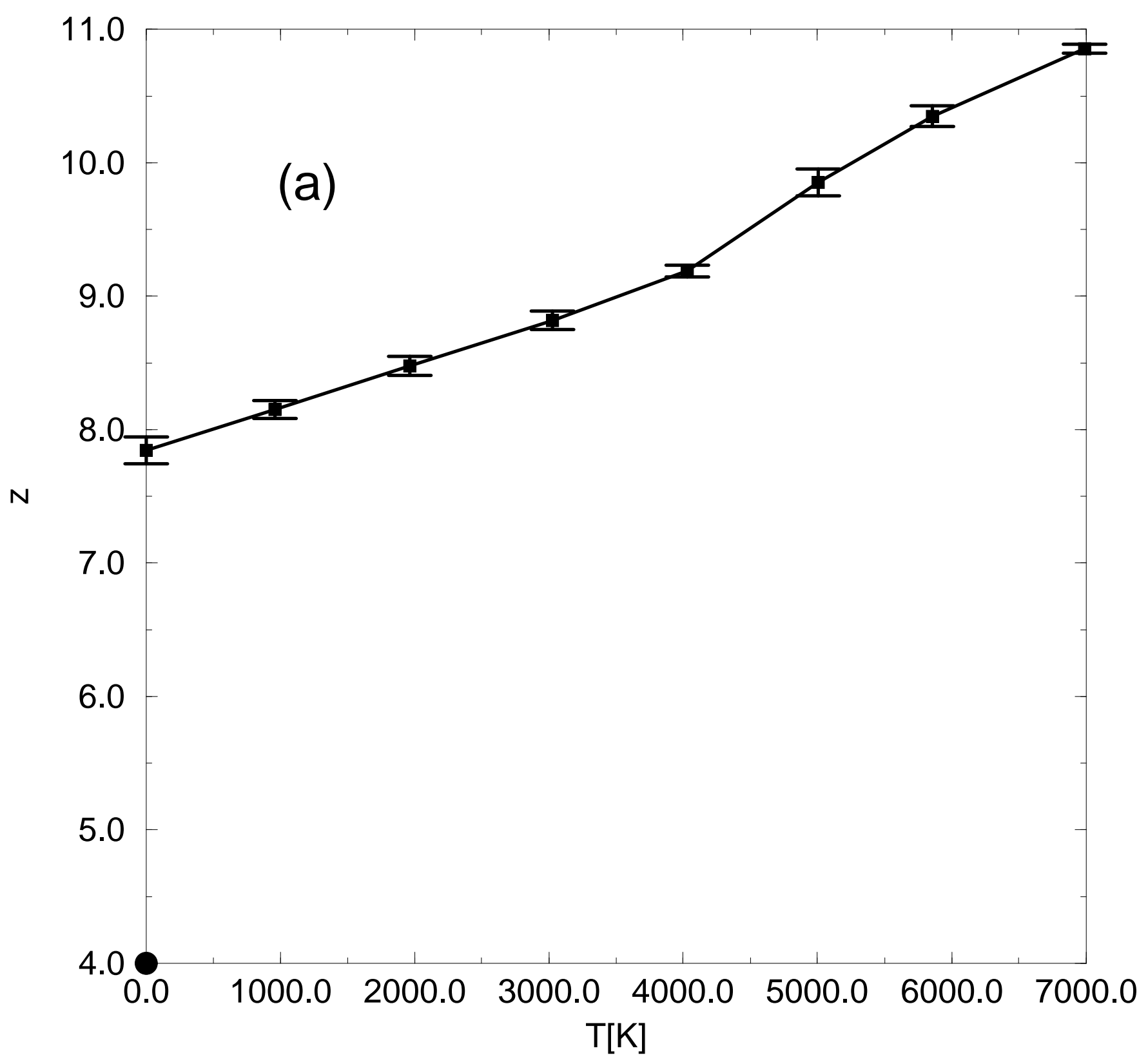




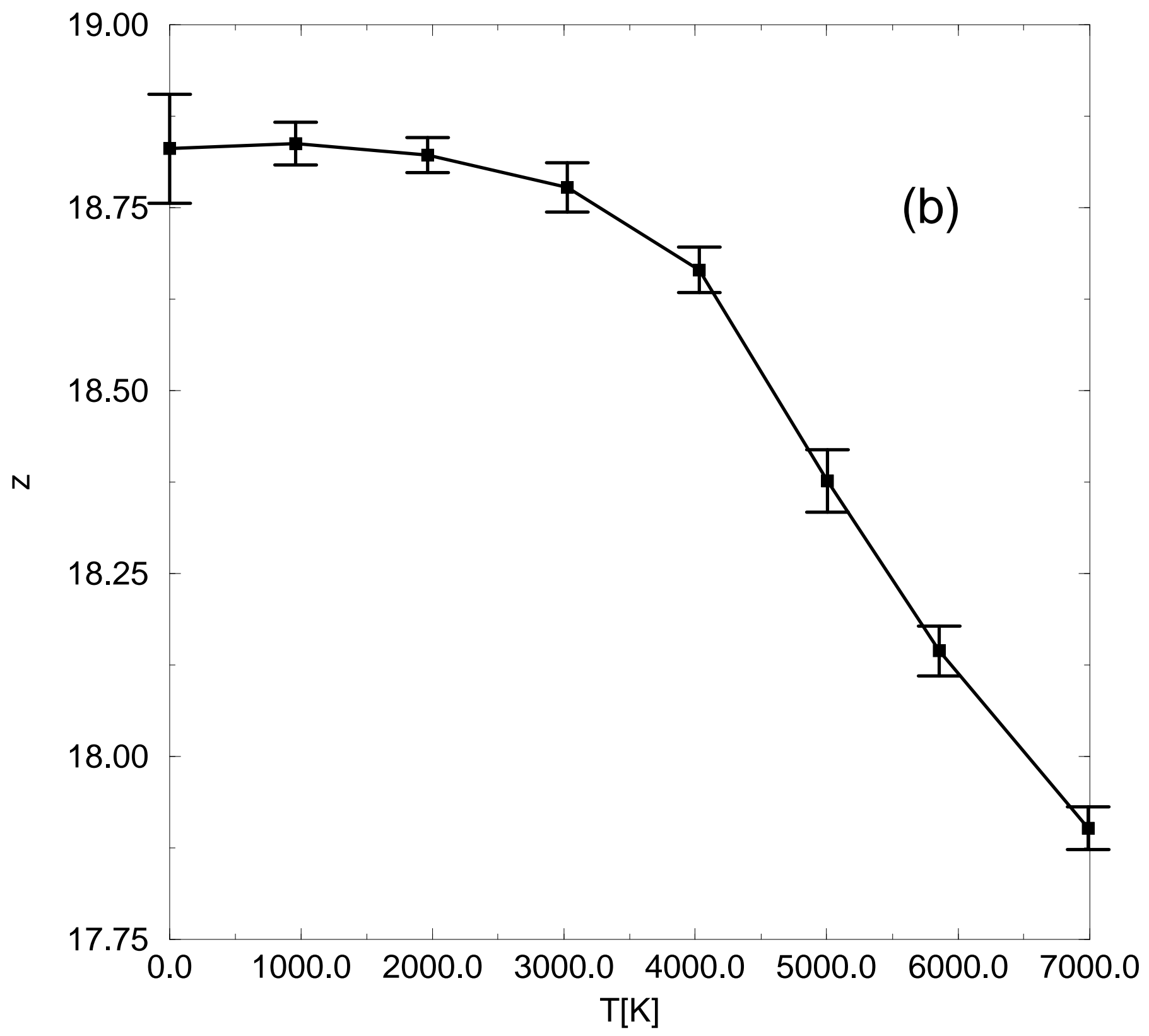


(a)

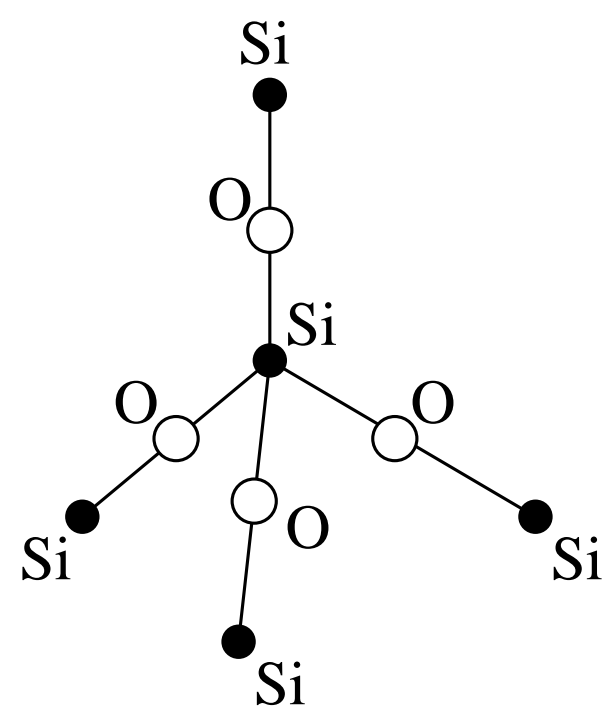

(b)

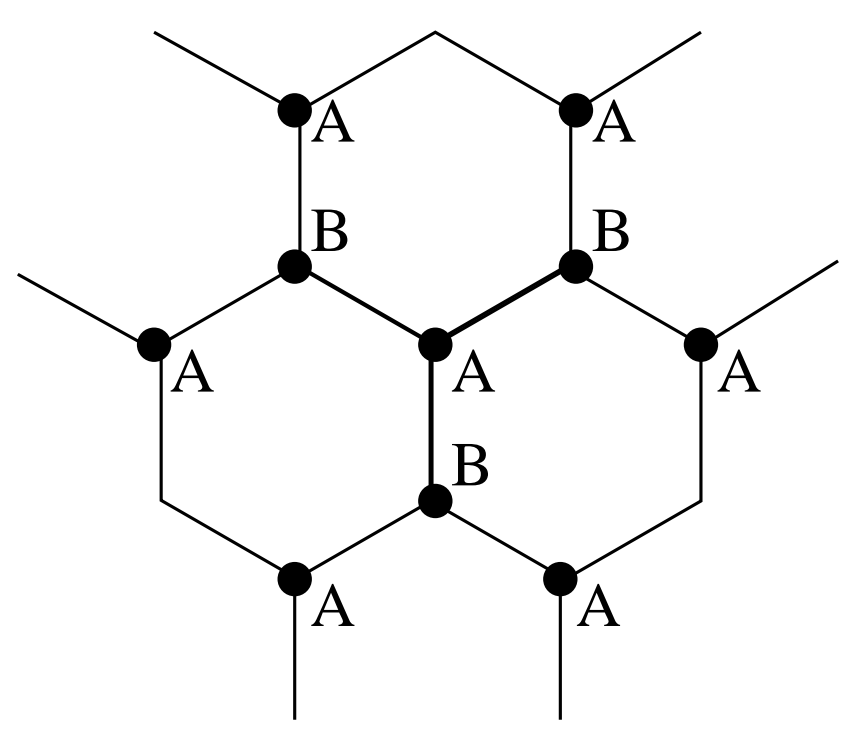

(c)

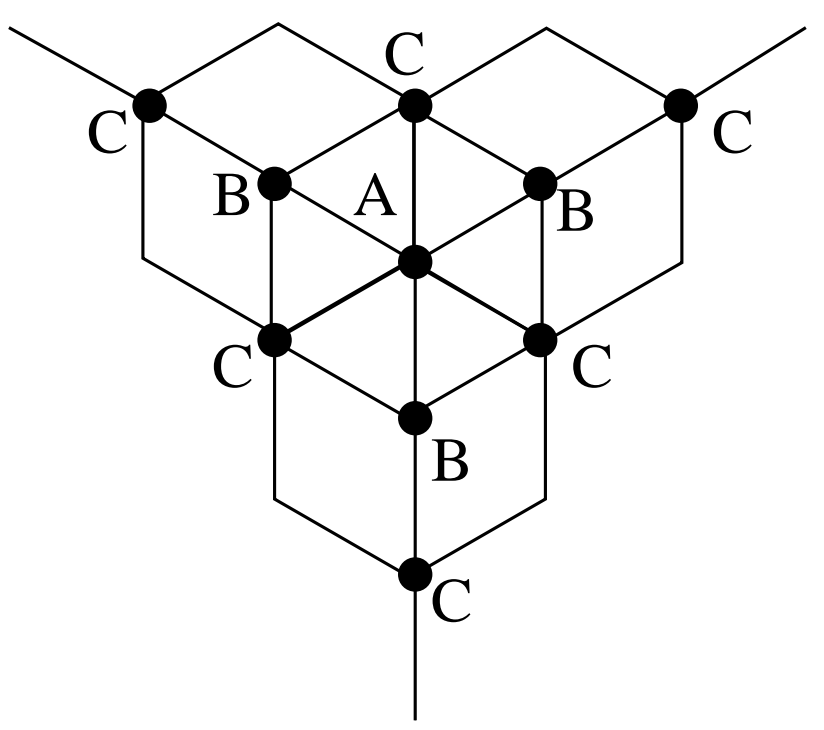

Figure 6 


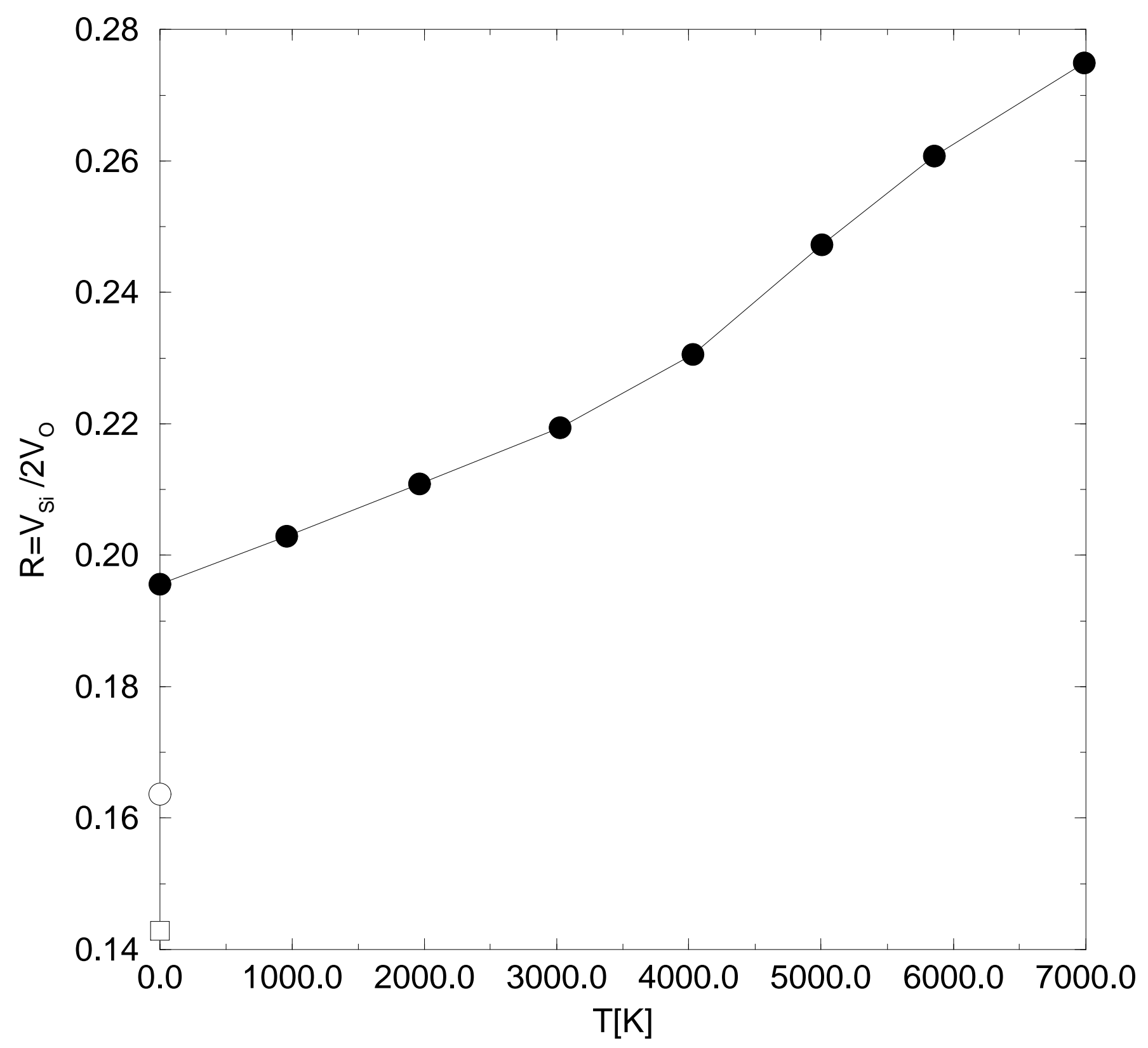

